\title{
INTERCAMBIOS, COMENTARIOS
}

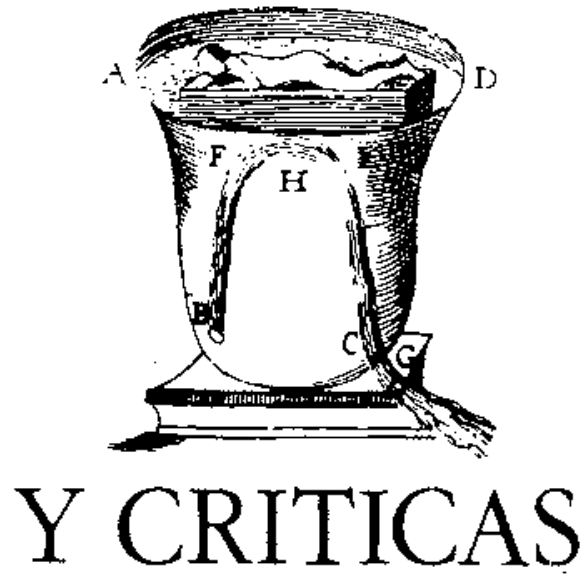

En esta sección intentamos recoger, por una parte, los comentarios y criticas sobre los trabajos aparecidos, asi como sugerencias de cualquier tipo que puedan contribuir a una mejora de la revista.

En segundo lugar pretendemos que estas páginas sirvan para dar a conocer la existencia de grupos de trabajo y facilitar asi los contactos e intercambios.

También pensamos que puede ser de interés el conocimiento de las lineas de trabajo seguidas por los distintos grupos, que pueden enviar breves resumenes de sus actividades.

Por ultimo contemplamos la posibilidad de favorecer los intercambios objeto de esta sección con la publicación de algunas entrevistas y mesas redondas.

\section{RELACION DE GRUPOS DE TRABAJO}

"SODLAC" CANaria (Sociedad para la Didáctica Integrada y Activa de las Ciencias)

La Sociedad Canaria para la Didác. tica Activa e Integrada de tas Ciencias "SODIAC" surge en el curso 1976-77 con los objetivos que transcribimos a continuación:

a) Elevar y actualizar el nivel profesional y pedagógico de los profesores de Ciencias.

b) Impulsar el desartififo de las investigaciones relativas a la Didáctica Activa e integrada de las Ciencias, así como preocuparse por su implantación en los Centros docentes.

c) Servir de nexo entre los profesores de Ciencias para intercambiar experiencias e ideas.

d) Gestionar ante los organismos competentes el apoyo necesario para la contribución a los fines anteriores.

e) Organización de cursillos y conferencias. publicación de revistas $y$ boletines $y$ cuantos medios contribuyan a la consecución de los fines anteriores.

En junjo de 1983 ha publicado un dossier de sus actividades que puede ser solicitado, al igual que cualquier otra infomación, a la siguiente dirección:

"SODIAC" CANARIA

C. N. Ramirez Bethencourt

Subida a San Francisco $5 / \pi$

Miller Bajo (Las Palmas)

\section{GRUPO DE QUTMICA DE LA} UNED

INTEGRANTES: $M^{a}$ Soledad Este. ban Santos. $M^{a}$ Rosa Gómez Antón

NIVEL EDUCATIVO: Universidad MATERIAS PUBLICADAS:

Unidades Didácticas de Quimica General

Unidades Didácticas de Quimica Orgánica

En tomo a la enseñanza de la Química en una Universidad a dis. tancia.
I. Análisis del rendimiento obtenido por los alumnos

II. Análisis de las variaciones en la matriculación.

PROYECTOS: Curso práctico de laboratorio para los alumnos de Universidades abiertas.

Ensenanza práctica de la Quimica con ordenador.

DIRECCION: Departamento de Quimica General y Macromoleculas Facultat de Ciencias. U.N.E.D Cuidad Universitaria. MADRID-3-

Hay sin embargo muchos casos. como por ejemplo los procesos redox en disoluciones no acuosas, o bien orgánicas complicadas, eл los que el método Ling puede resultar casi imprescindible. Podemos a' propio tiempo afirmar que este método da resultados óptimos en aque llos casos en los que los demás métodos de ajuste resultan dificiles o casi imposibles de aplicar. de suerte que, es precisamente en estas ocasiones donde su aplicación resulta 
particularmente

eficaz.

gratificante

y

gar al resultado deseado con la máxima rapidez, sencillez y eficacia.

\section{BIBLIOGRAFIA}

de ajuste ideal, adecuada para todos los casos. Debe pues, confiarse a la sensatez y buen hacer del usuario la eleccion del método que permita lle-
1. LING, J., "Easy Chemistry*, Vol. I. Goodman Publisher, Hong Kong, 1979 , p. 105-112.
2. SMITH, W. R. Y MISSEN, R, W. *What is stochiometry., Chem. Eng. Ed., (winter. 1979)

3. KENNEDY. I. H., "Balancing Chemical Equations with a Calcuiaton. J. Chem. Ed., 59, 6, 1982, p. 523.

KOLB, D.J., Chem. Ed., 58, 8, 1981, p 642.

\section{PRESENTACION DE LINEAS DE TRABAJO}

\section{RESUMEN DE UN MODELO DE TRABAJO PRACTICO PARA COU}

En los trabajos prácticos que están concebidos como pequeñas investigaciones se intenta seguir el esquema de un ciclo de investigación cientifica. Cuando se pretende seguir dicho esquema en trabajos de Química a niveles no elementales (tal es el caso de las experiencias que suelen proponerse en COU) se encuentran dificultades que solo pueden salvarse si se aplica el método con flexibilidad.

A continuación se propone un resumen de un srabajo práctico con objeto de presentar el método que puede seguirse. El trabajo consiste en la «Determinación de la fómula de un hidraton.

El primer caso consiste en presentar en clase el problema que piantean adgunas sales que contiene agua eliminable por calentamiento pero que presentan características diferentes a una mezcla sal-agua. A continuación se proponen cuestiones que permitan discutir las diferencias entre un compuesto y una mezcla, particularmente en el caso de una sal hidratada, así como los ensayos necesarios para verificar dichas diferencias. A modo de ejemplo se recogen aqui algunas actividades que podrian proponerse en esta etapa:

1. Cuando puede afimarse que una sal es un hidrato o por el contrario se trata de una mezcla, p. ej. sai hímeda?

2. ¿Qué ensayos habria que verificar para determinar si una sal es un hidrato?

3. ¿Cual seria la fórmula de un hidrato cualquiera? Aplicarlo al caso del sulfato de cobre (II) hidratado. Puede proponerse la resolución de las cuestiones anteriores en clase. Una vez discutidas se sugiere una actividad encaminada a resoiver un problema fundamental del trabajo: el diseño de las experiencias.

4. Diseñar un experimento para determinar la fórmula del sulfato de cobre (II) u otro hidrato cualquiera.

Sería deseable que la actividad anterior fuera resueita con tiempo después de pensarse suficientemente, o que puede proponerse para casa. Importaria conocer en primer lugar qué medidas interesaría efectuar para conocer la fór. mula del hidrato y en $2^{\circ}$ lugar se discutiria todo el abanico de soluciones propuestas en orden a evaluar las dificultades de los procedimientos, dispositivos o aparatos, grado de imprecisión, posibilidades de ertor que entraña el método, etc.

Una vez establecido el procedimiento experimental. cuya forma definitiva sería sugerida por el profesor, se procederia a la realización experimental, introduciendo cuestiones relacionadas con dicha readización, tales como la actividad que aquí se sugiere en 5 .

5. ¿Cuándo puede asegurarse que se ha eliminado toda el agua del hidrato?

Las siguientes actividades proponen la resolución del problema, p. ej.:

6. ¿Qué cantidad de agua se ha eliminado? ¿Qué cantidad de sulfato de cobre (II) antidro hay en la muestra? Calcular la fórmula del hidrato.

Pueden sugerirse además otras actividades como rehidratar la sal para observar el cambio de color, dejar a la intemperie un tiempo prolongado y volver a pesar. etc. A continuación se pasaría a la interpretación de los resultados

La última etapa sería el análisis de los resultados obtenidos por los diferentes grupos de alumnos que realizan silumtáneamente la experienia y confeccionar en el cuaderno de trabajo un guión conde figuren todas las actividades propuestas, las discusiones de sus soluciones y los resultados y conclusiones obteridos.

José Miguei AYENSA ESPARZA Instituto de Bactillerato CALAHORRA (La Rioja)

\section{MATEMATICAS:}

DVVESTIGACION SOBRE LA ENSENAANZA DE LOS ANGULOS

Al pretender enseñar el concepto de ángulo, desde un punto de vista supe. ror, a estudiantes de primeros cursos de Facuitades o Escuelas Universitarias, el profesor se tropieza con una variada gama de definiciones que, bajo la misma palabra «ángulo», estan dando conceptos diferentes (ángulos orientados, ángulos no orientados, media de ángulos).

El estudio de los ángulos empezó siendo un simple trabajo personal en donde mi única intención era hacer una exposición clara sobre el tema. 